\title{
Conceptualización y reflexión sobre la práctica educativa en un programa de formación continua para docentes de educación media superior en México*1
}

\section{Conceptualization and Reflection on Educational Practice in a Continuing Education In-Service Training Program For Teachers Of Higher Secondary Education In Mexico ${ }^{2}$}

\author{
Guadalupe Nancy Nava-Gómez ${ }^{3}$ \\ Universidad Autónoma del Estado de México \\ Instituto de Estudios sobre la Universidad \\ Toluca, México \\ ngnavag@uaemex.mx \\ Jenaro Reynoso-Jaime ${ }^{4}$ \\ Universidad Autónoma del Estado de México \\ Instituto de Estudios sobre la Universidad \\ Toluca, México \\ jreynosoj@uaemex.mx
}

Recibido: 2 mayo 2013 Aceptado: 4 diciembre 2014 Corregido: 30 diciembre 2014

Resumen: El artículo expone un acercamiento a la conceptualización de la práctica educativa en un programa de posgrado en la Universidad Autónoma del Estado de México (UAEM). A partir de la diferencia

1 La investigación que se presenta es un producto del proyecto de investigación titulado: "Diagnóstico sobre las condiciones reales de formación y transformación de la práctica educativa en el nivel medio superior: el caso de los estudiantes de la Maestría en Práctica Educativa para el Nivel Medio Superior (MPENMS)", registrado con la clave 3315/2012CHT. El proyecto recibió financiamiento de la Universidad Autónoma del Estado de México a través de la Secretaría de Investigación y Estudios Avanzados. El objetivo central consistió en la indagación de manera amplia sobre las problemáticas retos y actuales recurrentes en la implementación de un programa de estudios avanzados que coadyuvara a los esfuerzos por la profesionalización de los docentes del nivel medio superior.

2 The investigation presented is a product of the study entitled "Diagnosis on the actual conditions of formation and transformation of educational practice of high school teachers: A study case of the Masters in Educational Practice for High School Teachers", registered with the number 3315 / 2012CHT. The project received funding from the Autonomous University of the State of Mexico through the Secretary of the Research Studies. The main objective was to inquiry broadly about the current challenges and recurring problems in the implementation of a program of advanced study that would contribute to efforts to professionalize high school teachers.

3 Doctora en Educación Bilingüe por la Universidad de Texas A \& M-Kingsville, Texas, USA. Actualmente colabora como investigadora en el Instituto de Estudios de la Universidad de la UAEMéx. El campo de investigación al que pertenece es Ciencias de la Educación y Políticas Lingüísticas. Ha impartido cursos de investigación educativa, aprendizaje y adquisición de idiomas, sociolingüística, didáctica de lenguas, intervención educativa, entre otros cursos. De las publicaciones más recientes destacan las siguientes: The Impact of Regional Differences on Elementary School Teachers' Attitudes Towards Their Students 'Use of Code Switching in a South Texas School District, publicado en PROFILE Vol. 14, No. 1, April 2012. ISSN 1657-0790. Bogotá, Colombia; Nava Gómez, Guadalupe Nancy. (2011). Reseña de "SUBTRACTIVE SCHOOLING" de Ángela Valenzuela. Tiempo de Educar, Julio-Diciembre, 301-308; Home Storybook Reading and the Development of Linguistic Abilities During the Acquisition Stage in Spanish-speaking Children publicado en Journal of Border Educational Research (septiembre, 2011), vol. 10 (1); Análisis de la cultura lectora en alumnos universitarios (2009) publicado en la Revista Educación 33(1), 39-57, ISSN: 0379-7082, 2009 y La alfabetización en México: ¿Una realidad educativa o un problema cultural?, mismo que se presenta como capítulo en el libro: La complejidad multicultural Indoamericana, cultura etnicidad y derechos (En Massé\& Pedroza, 2011) ISBN: 9786077946045.

4 Coordinador del programa de Maestría en Práctica Educativa del Nivel Medio Superior. Investigador del Instituto de Estudios Sobre la Universidad (IESU) de la Universidad Autónoma del Estado de México. Publicaciones recientes: artículo: "Un acercamiento al sustento teórico de las competencias en educación básica“, Memoria del Segundo encuentro de la Red Nacional de Profesionales de la docencia y la difusión de la Historia, 2011, libro: Facciones políticas y elecciones en el Estado de México (1919-1926), México, IESUUAEM, 2011, artículo: "Vida, revolución y maestros" en Al pie de la letra, revista de la Escuela Normal de Tenancingo, año 6, No. 9, Enero 2012, artículo: "Complejidad y retos en la educación histórica por competencias”, Memoria del Tercer Encuentro Nacional de Docencia, Difusión y Enseñanza de la Historia y Primer Encuentro Internacional de Enseñanza de la Historia, septiembre 2012. 
entre programas educativos orientados a la profesionalización docente, se contextualiza la problemática sobre los programas actuales orientados a la formación de los docentes del nivel medio superior. El objetivo central del documento consiste en presentar experiencias pedagógicas de un programa de estudios avanzados, cuyo objetivo es la profesionalización de la práctica educativa, a partir de la re-significación de la práctica docente de los actores. Aspectos tales como actualización, calidad educativa y capacitación permanente son discutidos ampliamente desde el marco de las reformas actuales en materia educativa bajo el enfoque de una pedagogía crítica desde la investigación-acción. Como resultado, se argumenta que en México es necesario impulsar programas educativos de estudios avanzados, cuyo objeto de estudio sea la profesionalización de la práctica educativa. Asimismo, se sustenta que la actualización permanente de los docentes debe dejar de asumirse en forma de cursos cortos, talleres y seminarios, para dar paso a una formación continua y permanente de los docentes del nivel medio superior.

Palabras clave: México, práctica educativa, programas de formación docente, formación inicial, formación permanente, pedagogía crítica.

\begin{abstract}
This article presents an approach toward the conceptualization of the educational practice in an in-service teaching program in the Autonomous University of the State of Mexico (UAEM). After establishing the difference between initial and continuous educational programs, the problems about the current in-service teaching preparation programs oriented to prepare in-service teachers of higher secondary education are described. The aim of this paper consists of presenting pedagogical experiences of a continuous in-service teaching program whose objective is the professionalization of the teaching and educational practice based on the reconceptualization of it. Aspects such as the teacher updating, educational quality and permanent education are widely discussed from the current educational reforms in Mexico within a critical pedagogical framework. As a result, it is pointed out that Mexico requires promoting educational programs of higher education oriented to the professionalization of the educational practice. Additionally, it is argued that in-service teacher preparation must leave behind traditional practices such as short courses, workshops and seminars in order to enhance the continuous and permanent education of those teachers working at the higher secondary level.
\end{abstract}

Key words: México, educational practice, in-service teaching programs, initial education, continuing education, critical pedagogy.

\title{
I. Introducción
}

En México, el tipo de actualización y formación docente en nivel medio superior se reduce, generalmente, a cursos modulares cortos, diplomados y talleres. Por esta razón, se propone que, para contribuir al mejoramiento de la calidad de la educación, es importante el impulso a la actualización y formación permanente de los educadores a través de programas de estudios avanzados, cuyo objeto de estudio sea el saber de los docentes, fundamentalmente. Como un ejemplo, en los últimos cuatro años la UAEM ha optimizado la efectividad de las estrategias educativas de formación y profesionalización de los docentes con la finalidad de mejorar las condiciones del proceso de enseñanza-aprendizaje.

De 2005 a 2012, en la UAEM se consolidaron 122 programas educativos en nivel 1 de los Comités Interinstitucionales para la Evaluación de la Educación Superior (CIEES). Además, se aumentó a 73 programas educativos acreditados por organismos acreditadores reconocidos por el Consejo para la Acreditación de la Educación Superior (COPAES) (Gasca, 2012). El mayor número de programas acreditados se concentra en los organismos académicos. 
En 2012, 4 licenciaturas fueron reconocidas en el Padrón de Programas de Licenciatura de Alto Rendimiento Académico del Centro Nacional de Evaluación para la Educación Superior (CENEVAL): "tres con nivel 1: Ingeniería Civil, Químico Farmacéutico Biólogo y Trabajo Social, el programa de Psicología tuvo el nivel 2" (Gasca, 2012, p.17). A pesar de estos resultados, uno de los retos principales, tanto a nivel superior como medio superior, consiste en lograr que el $100 \%$ de los docentes cuente con una actualización y una formación profesional permanente.

El presente trabajo apunta a la conceptualización y reflexión de la práctica educativa a través del análisis e interpretación de las narrativas y testimonios de los estudiantes del programa de Maestría en Práctica Educativa del Nivel Medio Superior, en torno a sus representaciones y significaciones sobre su quehacer docente. Del cruce de estas miradas intentaremos conceptualizar la práctica educativa como objeto de estudio, recuperando la redimensión de los contextos y problemáticas educativas para comprender y diseñar estrategias efectivas y así abordarla desde la formación permanente del profesorado del nivel medio superior. ${ }^{5}$

\section{Antecedentes}

Como resultado, una de las estrategias que fue implementada como parte de los programas de mejora continua al proceso de formación profesional en la UAEM es el programa de Maestría en Práctica Educativa del Nivel Medio Superior, el cual fue creado con el propósito de profesionalizar la práctica docente de los profesores de este nivel educativo. Dicho programa atiende, actualmente, a una matrícula de 30 estudiantes de los cuales el $70 \%$ se trata de profesores de la UAEM, mientras que el $30 \%$ restante son profesores activos laboralmente en el Valle de Toluca, pertenecientes a subsistemas escolares tales como CBTIS (Centro de Bachillerato Tecnológico, Industrial y de Servicios), COBAEM (Colegio de Bachilleres del Estado de México), CONALEP (Colegio Nacional de Educación Profesional Técnica) (UAEM, 2010). Ante la necesidad de profesionalizar la práctica de los docentes, el programa de Maestría en Práctica Educativa, para el año 2015, estará funcionando como una opción más de formación continua para el personal académico de los diferentes subsistemas educativos. La propuesta implementada y la prospectiva de su operación presentan a la UAEM como una institución de educación superior responsable de la profesionalización de los docentes, lo cual resulta una condición para asegurar la calidad de la educación media superior y superior.

\section{Revisión de la literatura seleccionada}

\section{Actualización docente y formación permanente}

La formación y actualización docente representa un eje transversal y pieza fundamental para la proyección del futuro de la enseñanza en la UAEM. La calidad de la educación, en

\footnotetext{
5 La Universidad Autónoma del Estado de México, a través del Instituto de Estudios Sobre la Universidad (IESU), ofertó a partir de 2010 la Maestría en Práctica Educativa del Nivel Medio Superior con el objetivo de actualizar a los docentes y gestores de ese nivel educativo en la tendencia pedagógica de la educación por competencias, bases teóricas sobre las cuales se realizó una reforma a ese nivel educativo (UAEM, 2010).
} 
todos sus niveles, depende en gran parte de la actualización y formación permanente de los docentes. Narváez y Cadena (2009) exponen la problemática de la profesionalización de la práctica educativa en las universidades ya que, si bien la mayoría de los docentes que conforman los claustros cuentan con una formación especializada en las diversas áreas del conocimiento, por su formación profesional no están capacitados en los campos relacionados con la didáctica o la pedagogía; al respecto, los autores subrayan que:

Resulta, entonces, que los niveles de especialización alcanzados en el dominio de los objetos de conocimiento son los que acreditan finalmente el potencial y la capacidad de un profesional como docente universitario. Algunos podrían pensar que justamente esa experiencia, tanto en investigación como en prácticas académicas de posgrado, representa un saber suficiente para asumir responsabilidades docentes. (p. 57)

En este sentido, Elena Luchetti (2008) establece la diferencia entre la formación inicial y la formación continua. El primer tipo lo describe como la capacitación docente que la mayoría de los profesores posee, tal es el caso de las licenciaturas en pedagogía, ciencias de la educación y educación, fundamentalmente; esta categoría es de vital importancia ya que explica por qué en Latinoamérica se observa al interior de las instituciones el problema sobre la preparación no especializada del personal académico. Cabe señalar que, en contextos universitarios, esta primera modalidad de formación no excluye áreas del conocimiento tales como las humanidades, ciencias de la salud, ciencias duras y ciencias sociales, ingenierías y otras áreas donde se aborda el conocimiento humano como parte de planes de estudio de la educación terciaria.

No obstante, la formación inicial, si bien es importante, no es suficiente, ya que las necesidades actuales en el campo educativo exigen, por una parte, la actualización de los docentes y, por la otra, la especialización en las diferentes áreas del conocimiento que imparten al interior de las escuelas e instituciones académicas. Luchetti (2008) se refiere a esta categoría como la cenicienta de la educación latinoamericana, pues la formación inicial contribuye al desarrollo e instrumentación de programas educativos de manera significativa; sin embargo, no garantiza el logro de la calidad educativa o certificación de los programas académicos. Más aún, se requiere de la formación continua de todos aquellos que se encuentran frente a un grupo y cuya profesión les exige la actualización permanente en las distintas áreas del conocimiento que se contemplan al interior de los diferentes planes de estudio.

En contraste, el segundo tipo de capacitación docente se define como la formación continua o permanente, traducida en programas de grado y posgrado, cuyo objetivo es la profesionalización de la práctica docente, fundamentalmente, seguida de los programas de posgrado centrados en la investigación en las diversas áreas del conocimiento. Los objetivos centrales de este tipo de formación son la capacitación, la actualización y el perfeccionamiento de la práctica docente a través de programas educativos de actualización en las diferentes disciplinas. Para este fin, Luchetti (2008) recomienda dejar de asumir este tipo de formación docente, en su modalidad de cursos cortos presentados de manera desarticulada, para "romper con la lógica de sumatoria de cursos y generar una transformación de las prácticas educativas en las instituciones" (p. 14). 


\section{La práctica educativa vista desde la pedagogía crítica}

El objeto de estudio del programa de Maestría en Práctica Educativa del Nivel Medio Superior consiste en analizar la práctica educativa una vez que ésta es entendida como la acción político-pedagógica que ejercen las distintas comunidades para formar a sus ciudadanos (Freire, 1996, p. 21). A la luz de esta propuesta, Freire señala que en el ejercicio le parece fundamental "dejar claro desde el inicio que no puede existir una práctica educativa neutra, no comprometida, apolítica" (p. 42). En todo momento, se sugiere que la práctica educativa esté orientada hacia un objetivo o fin específico; jamás encaminada a la neutralidad. Para lograr lo anterior, los profesores deberán asumir, con compromiso y responsabilidad, su papel como agentes activos partícipes en las transformaciones a partir del análisis y reflexión de su práctica.

En este sentido, las posibilidades que ofrece la UAEM para contribuir con el proyecto nacional en materia de formación docente son altas. Por ello, proyectos educativos similares se van sumando a los esfuerzos de profesionalizar la práctica educativa, lo cual garantiza la cobertura y la calidad de los programas. Cabe señalar que la instrumentación de este tipo de planes de estudio, tales como la Maestría en Práctica Educativa del Nivel Medio Superior, requiere de cambios profundos en la cultura de los docentes del nivel medio superior que les permita reflexionar sobre el significado de las actividades que constituyen su práctica educativa, para comprender el papel que desempeñan dentro de sus espacios escolares, y, en este sentido, se dé el último paso hacia la docencia crítica y reflexiva de manera democrática e incluyente. A decir de Luchetti (2008), "se requiere, ante todo, estar francamente dispuesto a revisar la matriz con la cual uno aprendió, los esquemas, los prototipos, los modelos internos (...) estar dispuestos a abordar una reestructuración intelectual” (p. 14).

Esta praxis involucra un marco donde se ubica la labor y la participación docente más allá de un método por seguir o una técnica por aplicar; implica un cambio de fondo en la cultura de los docentes del nivel medio superior. Este cambio conlleva a la construcción de una visión innovadora de educación incluyente y participativa en donde cada uno de los integrantes de las instituciones educativas tiene algo que decir y algo que hacer. En este último aspecto, el docente asume la responsabilidad de formarse de manera crítica, continua y reflexiva, pero principalmente el reconocimiento de que se requiere de un proceso de actualización en su campo disciplinar y su saber docente (Giroux, 1990; Freire, 1996, 1997). Por las razones aquí expuestas, en la actualidad ya no es suficiente con estar habilitado en uno de los campos del conocimiento; no basta con estar acreditado como docente si dicha acreditación ocurrió en momentos distintos a las circunstancias vigentes que operan en las diferentes instituciones.

Como punto coyuntural, el docente del nivel medio superior debe contar con una formación profesional sólida que le permita participar directamente en los distintos procesos de mejora y toma de decisiones que acontecen en torno a la práctica educativa. Desde el punto de vista de la pedagogía crítica, Freire (1996) sugiere lo siguiente:

El mejoramiento de la calidad de la educación implica la formación permanente de los educadores. Y la formación permanente se basa en la práctica de analizar la práctica. Es 
pensando su práctica, naturalmente con la asistencia de personal altamente calificado, como se puede percibir integrada en la práctica una teoría no percibida antes, poco percibida o ya percibida pero poco asumida. (p. 81)

En consecuencia, resulta imperiosa la necesidad de significar la propia práctica para identificar las fortalezas y debilidades que pudieran influir en el éxito o fracaso de nuestra enseñanza. Lo anterior será posible siempre y cuando el docente asuma la crítica, ésta entendida como el reconocimiento, ya sea total o parcial, de que "se estaba incurriendo en un error que merecía ser corregido o superado" (Freire, 1996, p. 65). Sin embargo, este proceso requiere de una transformación de las prácticas actuales de enseñanza centradas en la continuidad de modelos individuales de los que nos vamos apropiando durante nuestro paso por las escuelas. La enseñanza, en gran medida, depende del conocimiento empírico que conduce a la generación de patrones a seguir en razón de presentar contenidos a los educandos. Por lo tanto, la forma en la que nos presentamos como docentes incluye las experiencias previas que tuvimos como estudiantes, pero no necesariamente conduce hacia la reflexión y la crítica como parte del análisis del quehacer docente.

Por otra parte, Pedroza (2011) explica que el cambio y la mejora educativa pueden lograrse a través de la investigación-acción y sugiere que el punto de partida es la práctica de los docentes, la cual posteriormente deriva en la construcción de una teoría. De esta manera, se tendría que la reflexión en la práctica es la vía a través de la cual los docentes pueden aproximarse a la teoría. En la presentación de su libro La investigación-acción en la práctica educativa-reflexiva, la propuesta central del autor se resume de la siguiente manera:

El método elegido, parte de la práctica sin olvidar la teorización, procede por círculos que son cualitativamente diferentes en la medida en que se avanza en espiral. En este sentido, se aporta el supuesto que la práctica y la teoría van siendo cualitativamente diferentes conforme se avanza en el proceso de la investigación-acción de la práctica educativa. Propongo un modelo de investigación-acción acorde a los cambios en la práctica y en la teoría. Que dan cuenta de tres saltos cualitativos en la profesionalización de la práctica educativa: la significación, la intervención y la re-significación (Pedroza, 2011, p. 7)

En la cita anterior, el autor señala que la significación de la práctica educativa conlleva a que el docente se transforme en investigador crítico y reflexivo de su propio quehacer. A decir de Giroux (1990), se requiere repensar al docente como un intelectual conocedor e investigador de su propia praxis. Es precisamente ésta la orientación que tiene el programa de Maestría en Práctica Educativa del Nivel Medio Superior, la profesionalización de la práctica de los docentes a partir de la articulación entre la enseñanza crítica desde el marco metodológico de la investigación-acción. En este sentido, la finalidad de la práctica educativa consiste en posibilitar la enseñanza de contenidos a las personas, por una parte; y por otra, concientizarlas (Freire, 2009). Adicionalmente, se recupera la necesidad de desarrollar programas de estudios avanzados desde una visión de la pedagogía crítica, en la cual el sujeto logre comprender su posición dentro de su contexto, lo cual implica su participación, su integración y su representación objetiva de la realidad. Cuanto más se hable de reformas 
educativas, mayor será la necesidad de procesos educativos que se desarrollen y gesten desde el interior de las instituciones de educación superior. Lo anterior, se genera como parte del trabajo educacional, lo cual sobrepasa los límites estrictamente pedagógicos, ya que se requiere tanto de una formación para la decisión y la participación como una para la responsabilidad social y política de los ciudadanos (Freire, 1997, 2009).

\section{Descripción de la problemática}

Tal y como se señala en las primeras páginas, la actualización de los docentes, en gran medida, ha sido realizada hasta el momento a través de talleres, cursos y diplomados organizados por módulos. Por esta razón, resulta difícil entender su articulación en función de ser considerados o revalidados como parte de estudios de posgrado. Hasta el momento, en México no existen mecanismos de transferencia o revalidación de estudios que les permitan a los profesores dar una continuidad a este tipo de formación profesional, pues su puesta en práctica es coyuntural y obedece a políticas educativas de corta visión. Otro aspecto relacionado con los cursos y diplomados es su caducidad o vigencia de ese tipo de actualización y formación docente. Esta situación es ampliamente discutida entre los docentes del nivel medio superior, quienes ante la necesidad y, en ocasiones, ante las exigencias de sus instituciones se ven obligados a elegir cursos temporales como parte de sus periodos inter-semestrales con la finalidad de obtener un documento que se traducirá en puntos para los diferentes programas de estímulo a los docentes o bien que les garantizará su permanencia en sus distintos espacios escolares. A pesar de los esfuerzos de las diversas instituciones por analizar el tipo de capacitación que requieren los docentes, aún se observan rezagos importantes en materia de la profesionalización de la práctica educativa.

De las ideas anteriores se derivó una pregunta de investigación, cuyos conceptos centrales sirvieron para realizar el análisis de las narrativas de los docentes de educación media superior expresadas a través de los distintos productos académicos y reflexiones que se les solicitaban en sus diferentes asignaturas que cursaban. Los participantes del estudio se convirtieron transitoriamente en estudiantes del programa de posgrado, como resultado de la reforma del bachillerato por competencias; ${ }^{6}$ la interrogante científica se propuso conocer: ¿Cuáles eran los conceptos de práctica educativa que los estudiantes de posgrado podían abstraer durante el trayecto formativo al ser estimulados con actividades académicas, cuyo objetivo central era el ejercicio del pensamiento reflexivo?

\section{Método}

El alcance de esta investigación es de naturaleza exploratoria e interpretativa (Borg, 1998; McEwan y Egan, 1998; Pruzo, 2002a, 2002b). Los estudios interpretativos en el campo

$6 \quad$ El enfoque de la educación por competencias se ha impuesto en el sistema educativo mexicano. Las primeras reformas se llevaron a cabo en los colegios de educación técnica en la última década del siglo XX, luego se impulsaron cambios en la educación superior de las universidades y tecnológicos, posteriormente se modificó la educación básica: preescolar en 2004, secundaria en 2006, primaria en 2009, la cual quedó articulada bajo ese enfoque en 2011 y, finalmente, en 2008 se llevó a cabo la reforma integral a la Educación Media Superior (RIEMS) a partir del acuerdo 444 de la Secretaría de Educación Pública (SEP), el cual estableció las competencias constituyentes de un marco curricular común en torno al cual se creó el sistema nacional de bachillerato (SEP, 2008 p.1-2). 
contemplan la participación de los investigadores en tiempos y escenarios naturales, lo cual abona a la especificidad del contexto y su cotidianeidad. Posteriormente, se realiza un ejercicio de reflexión analítica sobre lo revelado en el ambiente natural donde se desarrolla la práctica de los docentes del nivel medio superior a fin de elaborar un informe que incorpore el conocimiento específico y los significados de los docentes, esto es, su saber docente. A decir de Erickson (1997), éste último también es referido como el conjunto de significados locales que se traducen en parámetros universales de comportamiento. En el ejercicio de implementación del programa de estudios avanzados de Maestría en Práctica Educativa del Nivel Medio Superior, resulta importante llevar a cabo un acercamiento hacia los docentesestudiantes con la finalidad de identificar cuál ha sido el proceso de apropiación de una formación encaminada al reconocimiento de su propio quehacer docente. Asumir la práctica educativa conlleva una transformación individual con repercusiones en el plano social. Por ello, se recopiló información a través de sus narraciones que desarrollaron como parte de los productos académicos. Los productos académicos consultados para este estudio se encuentran albergados en la comunidad de aprendizaje, Formación basada en competencias, respaldados en la plataforma educativa SEDUCA ${ }^{7}$, propiedad de la UAEM.

\section{Pensar el concepto de la práctica educativa: La experiencia}

Paulo Freire $(1996,1997)$ define la práctica educativa como la participación democrática en la construcción de la ciudadanía. Sin embargo, esta categoría resulta en amplio grado opuesta a la concepción que los docentes de educación media superior tienen sobre su función en el espacio escolar. Las condiciones laborales de la gran mayoría de los profesores se caracterizan por exceso de horas clase, atención a varios grupos con un número importante de estudiantes, entre 45 y 50 por grupo, y en ocasiones distancia y tiempo reducido para traslados de una escuela a otra, ya que los docentes deben cambiar de espacio de trabajo a fin de cumplir con los contratos que les permitan obtener ingresos suficientes y una situación de vida decorosa.

Las condiciones de trabajo de los docentes del nivel medio superior tiene una correlación directa con las posibilidades de revisar el concepto de su propia práctica por lo que, cuando, saturados de actividades y angustiados por el ingreso económico tienen que desarrollar actividades de formación continua, la experiencia de práctica educativa que vivieron durante su tránsito como estudiantes es más fuerte y se impone al desarrollo de sus prácticas en los salones de clase y en sus diferentes roles al interior de los centros educativos.

De una suerte de combinación de la situación laboral, de la presencia sólida de los modelos pedagógicos experimentados y de las inercias didácticas que la institución impone como obligatoria a los docentes, resulta un limitado margen de maniobra intelectual para reconocer el tipo de interacciones que componen la práctica educativa. Como resultado de lo anterior, se observa una concepción limitada del trabajo cotidiano de ser profesor de bachillerato: presentación y evaluación

$7 \quad$ Portal de Servicios Educativos a Distancia de la Universidad Autónoma del Estado de México (SEDUCA), cuya dirección electrónica es: http://www.seduca2.uaemex.mx/. Ahora bien, los productos académicos se encuentran localizables siempre y cuando la persona sea un usuario registrado en la siguiente dirección electrónica: http://www.seduca2.uaemex.mx/distancia/Portafolio/portafolio.php?i $\underline{\text { Com }=10388 \& i \operatorname{Int}=136314 \& i C v e E s t=}$ 
de los contenidos básicos de cada curso que se imparte. De esa manera, la posibilidad de conceptualizar la práctica en un sentido más amplio, en el marco teórico que define el curriculum reformado o desde una postura crítica alternativa, se convierte en un fuerte reto para aquellos quienes se inscriben como estudiantes del posgrado en práctica educativa.

Visualizar la práctica educativa en el sentido freireano, con fines de construcción del ciudadano en el marco de una experiencia aúlica e institucional democrática, es un objetivo aún lejano en la práctica y reflexión teórica del profesor de bachillerato. La gama de expresiones que se observa en los productos académicos de aquellos quienes buscan superarse profesionalmente transita entre la preocupación por cumplir con las disposiciones administrativas, la angustia por comprender las nuevas categorías pedagógicas de las competencias genéricas o disciplinarias como base de una práctica educativa diferente, hasta la postura radical de rechazar los nuevos planteamientos curriculares. Todo ello, paradójicamente, según la opinión de los docentes participantes, sólo busca estimular la preparación de los estudiantes para el trabajo en las empresas capitalistas y dejan de lado una formación humana integral.

La respuesta a la actividad intencional de que los profesores, y al mismo tiempo estudiantes de posgrado, conceptualizaran su práctica docente es heterogénea, pues se encontraron dificultades para comprender los argumentos y verbalizarlos para esbozar un concepto parafraseado de tal manera que se recurre constantemente a la cita textual y a pesar de ello no logra el propósito, como se puede detectar en el siguiente ejemplo.

La actividad consistía en leer el texto de Luis Gerardo Meza La teoría en la práctica educativa (2002) y elaborar un ensayo de cuatro cuartillas donde presentarían una reflexión clara y fundamentada de la posición del autor sobre el tema. En un apartado del ensayo debería escribirse el concepto de práctica educativa que planteaba el autor, considerando sus fuentes; en este, una estudiante escribió lo siguiente:

Kemmis, es (sic) prólogo de Carr, aclara el punto en efecto, que nos dice: 'Para el profesor en ejercicio, ¿puede haber alguna idea más inocente, más transparente, más familiar que la de "práctica"? Es lo que hacemos. Es nuestro trabajo. Habla por sí misma , o al menos es lo que pensamos alternativa o la práctica. Es una forma de poder; o cambio social, la cual está, en gran medida, en manos de los profesores. (García, 2012, p. 1)

En la cita se observan tres puntos centrales: En primer lugar, la dificultad para expresar coherentemente las ideas detectadas en las fuentes teóricas del texto. En segundo lugar, no se nota la intención de cumplir con el propósito y título del apartado, el cual consistía en definir la práctica educativa, sino que escoge un párrafo donde tiene mayor protagonismo la dificultad de aprehender la conceptualización. Finalmente, en tercer lugar, resalta en la cita una respuesta poco clara y argumentada a la pregunta, ¿qué es la práctica educativa? al señalar esquemáticamente que se trata del trabajo que hacen los docentes o de una forma de poder de éstos.

En un segundo producto de la misma actividad, se observa la capacidad de interpretar con seguridad el concepto al asegurar que "la práctica educativa se concibe como el conjunto de experiencias que resultan de los problemas y situaciones a los que se enfrentan los 
docentes en su papel de formadores y facilitadores del conocimiento"(Macedo, 2012, p. 2); aquí no sólo se evitan argumentos relacionados con problemáticas concomitantes del objeto a conceptualizar, sino que, con base en una idea clara, propone consecuencias de la relación práctica-teoría bajo el siguiente discurso:

Con el supuesto hecho de que "la práctica hace al maestro", muchos profesores dejan a un lado la teoría para dedicarse por completo a su práctica educativa, esto sin duda incrementa su experiencia y sus habilidades para lidiar con los problemas que surgen de dicha práctica, pero por otro lado les puede ocasionar dos graves problemas: en primer lugar, su práctica se vuelve individualista ya que son los únicos que tienen conocimiento de lo que acontece en su ambiente de trabajo y que le dan solución a los problemas que enfrentan en su actuar diario. En segundo lugar, pueden llegar a creer que lo saben todo y que no necesitan del conocimiento de una metodología y estrategias las cuales les pueden resultar imprácticas y descontextualizadas (Macedo, 2012, p. 3).

Como puede notarse, la conceptualización de la profesora ratifica indirectamente la sustancia de la práctica educativa en la experiencia y las habilidades que, para resolver los problemas de su ambiente de trabajo cotidiano, los docentes ponen en juego; sin embargo, logra destacar, a partir de la observación y contraste de su práctica con la de otros, las consecuencias de la falta de reflexión y acercamiento a la teoría educativa en la formación de maestros individualistas. También expone el peligro de asumir una postura anti-intelectual de quienes son los encargados de estimular la búsqueda y la producción de nuevos conocimientos.

En un siguiente nivel de problematización se encuentran los planteamientos, a propósito de las limitaciones que las actividades de actualización presentan, para lograr articular la teoría con la práctica y lograr la acción recursiva de ésta última con una nueva teorización. En esta dirección, una profesora reconoció haber asistido a varios cursos de actualización, talleres de planeación y de microenseñanza, donde los docentes asistentes se ponían en el papel de estudiantes para tratar de entender sus necesidades y problemáticas; sin embargo, señala que, aunque "es un buen ejercicio de práctica docente dista de ser auténtico porque por mucho que los profesores imiten a sus estudiantes nunca podrán saber con certeza el origen de sus problemas" (Macedo, 2012, p. 4). Lo que la profesora destaca como positivo de las reuniones capacitadoras es que los docentes comparten sus experiencias y eso puede ser una base tanto para tomar decisiones como para crear nueva teoría educativa que sirva para otros ámbitos.

Sobre las posibilidades de pensar el concepto de la práctica educativa, los docentesestudiantes viven la coyuntura que se creó con la Reforma Integral de Educación Media Superior (RIEMS, de ahora en adelante), la cual estableció un sistema nacional de bachillerato que unificó los distintos subsistemas de ese nivel mediante el establecimiento de un marco curricular de competencias genéricas, disciplinarias y profesionales (SEP, 2008). ${ }^{8}$ La mayoría

8 Como resultado de la reforma federal de Educación Media Superior (RIEMS), que impuso el enfoque de la educación basada en competencias, la UAEM firmó en agosto de 2009 un compromiso con la SEP para adherirse al Sistema Nacional de Bachillerato, por lo que se dio a la tarea de revisar su plan de estudios 2003 para adecuarlo el perfil de egreso que establecieron las competencias genéricas y profesionales, de lo cual surgió el Currículo del Bachillerato Universitario 2009, hoy vigente en los planteles universitarios de la escuela preparatoria. Inmediatamente comenzó la revisión de los programas de asignatura para alinear los cursos en la búsqueda del perfil de egreso que estableció la reforma para unificar los diferentes subsistemas de educación media superior en México. 
de los docentes ya cursó un diplomado sobre el enfoque por competencias que subyace al plan de estudios del que se desprenden las unidades de aprendizaje a su cargo, lo que significa que ya tienen conocimiento sobre el papel distinto que la docencia del desarrollo de competencias le asigna a cada uno de ellos. Como se sabe, la propuesta de que el acto educativo consiste en conducir el aprendizaje de contenidos, el desarrollo de habilidades y la formación de actitudes o valores, conlleva una transformación del papel y trabajo del docente y, por tanto, la toma de conciencia de la importancia de poseer las competencias propias del docente para, a su vez, conducir el desarrollo de las competencias en los estudiantes.

Entre los efectos positivos de la coyuntura establecida por la RIEMS, se detectó la generación de una polémica que recorre los espacios institucionales en los que los profesores se mueven y encontró, en las actividades establecidas en cada una de las unidades de aprendizaje de la maestría, una vía de reflexión y de expresión de las distintas posturas que se asumieron frente a la reforma curricular del nivel. La discusión que la reforma y las actividades de actualización habían despertado sobre la práctica educativa se vio favorecida con la lectura de textos especializados y la construcción de escritos derivados de la reflexión que la primera estimulaba.

A través de los escritos y productos académicos de los estudiantes se observa que una de las principales problemáticas que enfrenta la renovación de la práctica educativa, tiene que ver con el anclaje en el modelo que se busca desplazar. De esa manera una profesora de bachillerato aceptó que la reforma educativa tenía aspectos positivos; pero, señaló que "algunos docentes nos resistimos al cambio, creemos que en esas reformas subyacen otras intenciones por parte del sistema educativo y de nuestras autoridades educativas; otros, simplemente nos hemos arraigado tanto a nuestras viejas prácticas pedagógicas que nos suena a inestabilidad cualquier práctica que implique algún cambio ya sea en lo administrativo, pedagógico o didáctico"(Cruz, 2012 , p. 2). Así, la resistencia a una nueva concepción de práctica educativa se convierte en un muro infranqueable toda vez que quien se resiste asegura "que es una utopía pues al regresar a la realidad del aula se enfrentan con estudiantes que obstaculizan la puesta en marcha de las propuestas de la reforma, que nos dejen tranquilos porque de todas formas los estudiantes no responden a los cambios y muestran una actitud apática hacia el estudio" (Cruz, 2012, p. 3).

Con el objetivo de aprovechar la discusión sobre el nuevo enfoque curricular entre los docentes de bachillerato e insertarlos en la reflexión sobre la necesidad de conceptualizar su trabajo, como estudiantes se les preguntó lo siguiente: si notaban cambios significativos en cada uno de ellos o en sus compañeros de centro de trabajo; si se había dado ya un proceso de transformación de las habilidades docentes que demandaba el modelo educativo reformado; o si sólo se habían logrado pequeños cambios. Entre las respuestas se pudo observar una amplia franja de docentes que se ubica en la defensa de su formación, en su experiencia centrada en la enseñanza y en la obediencia fundamentalmente, así como poseedores del conocimiento que se debe transmitir a los estudiantes; los docentes estudiantes llegaron a calificar a sus compañeros como tradicionales por su tendencia a reproducir el discurso.

La defensa del modelo de práctica docente se expresa poco de manera abierta y fundamentada; más bien adopta características de adaptación para la sobrevivencia, como puede notarse en la siguiente narración de una estudiante de la Maestría en Práctica Educativa del Nivel Medio Superior: 
(...) es preciso considerar un aspecto que se denota en la formación docente, la tradición respecto de lo que significa ser docente, aún permea la idea de reproducir el discurso, sin pensar en su práctica docente, la práctica reflexiva no se manifiesta y la distancia entre teoría y práctica se expresa claramente así que sin duda ante el planteamiento, las habilidades que el profesor requiere no se han generado del todo, quedan enmarcadas en un documento expreso, se circunscriben como requisito para cumplir con la función de la práctica docente, pero al final todo se encuadra en lo que permanece y no se transforma (Romero, 2012, p. 2).

El diagnóstico del comentario anterior revela la adopción de un nuevo discurso por parte de los docentes para cumplir con la autoridad; pero, al mismo tiempo expresa la dificultad de llevar a la práctica nuevas formas de proceder congruentes con el ideal educativo. Lo anterior puede interpretarse como una respuesta estratégica de los profesores que se esfuerzan por adoptar el nuevo discurso como un paso previo para practicar un proceso de aprendizaje en el que ya no son el centro de atención. Comprender el enfoque por competencias y el concepto implícito de práctica educativa no ha sido fácil; sin embargo, los esfuerzos fructifican, como lo evidencia el siguiente texto:

La práctica cotidiana de la docencia encierra diversos procesos y habilidades encaminados a la transmisión, transferencia, apropiación y ejecución del conocimiento. Desde ésta (sic) perspectiva es prudente decir que el saber hacer, el saber ser y el saber valorar de los docentes debe estar aparejado con el saber hacer, el saber ser y el saber valorar de los estudiantes, de tal forma que las habilidades y herramientas que el docente posee van a ser el medio por el cual el estudiante se pueda apropiar del conocimiento (aprehender). (Romero, 2012, p. 3)

En el extracto anterior se observa ya la construcción de un concepto más complejo de la práctica educativa en el contexto del enfoque por competencias, pues al tiempo que se plantea una función distinta del conocimiento, ya no sólo su reproducción, se hace patente la necesidad de que el profesor disponga de amplio conocimiento y habilidades didácticas para lograr que sus estudiantes se lo apropien y apliquen, así como de una postura ética; es decir, del desarrollo y posesión de competencias propias de la profesión y práctica docente como condición para conducir a los estudiantes en la misma senda del desarrollo de competencias para enfrentar la vida.

\section{Propuestas para iniciarse en la práctica reflexiva y la formación continua}

Como parte del proceso inicial para la formación permanente de los docentes se requiere del análisis y evaluación sobre la propia práctica. Naturalmente, pensar la práctica deberá realizarse con la asistencia de personal altamente calificado que pueda ayudar a asociar la práctica significada de los docentes (Freire, 1996, p. 81). Esta capacitación puede otorgarse mediante programas de posgrado, cuyo objeto central de estudio es la práctica educativa, la cual incluye la práctica docente. Sin embargo, resulta quizá un ejercicio poco útil considerar la práctica educativa sin la participación directa de los docentes en los distintos procesos de transformación que ésta va teniendo, en el transcurso del tiempo, como respuesta a las 
reformas educativas. El proceso de cambio está lejos de evocar la pasividad de los docentes. Asumir la necesidad de cambio no proviene de señalamientos externos o políticas educativas en momentos históricos determinados; la acción de asumir implica la participación democrática y voluntaria como parte de la nueva cultura con los docentes y no sólo para ellos.

El programa de formación docente es el contexto en el que se pueden expresar las distintas posiciones que, respecto de la reflexión, se van despertando en los estudiantes y docentes en servicio. Por ejemplo, un docente con poco tiempo de servicio logra reflexionar, como resultado de las actividades de estudio, que su trabajo es difícil y colosal en tanto que implica desgaste físico, psicológico y demanda estar en constante búsqueda de conocimientos y técnicas educativas. Como resultado, asegura estar

Completamente convencido que el profesor actual debe estar en búsqueda de elementos que le permitan enfrentar las nuevas exigencias educativas que se le exigen, y de buscar la reivindicación de su papel en la sociedad. Lo anterior, lo (sic) logrará precisamente generando una reflexión sobre sí mismo y de su desempeño para generar los cambios en su práctica educativa (Mendoza, 2012, p. 1).

Al tiempo que reconoce el carácter arduo de la práctica docente, también observa lo complejo que resulta el hacer un autoanálisis de la vida y del trabajo propio. No obstante, el placer de enseñar, la emoción de aprender lo que se necesita saber para enseñar y la conciencia de que el país necesita de ayuda, lo estimulan para entrar en un proceso de auto reconocimiento de lo que no funciona. Al respecto, señala lo siguiente:

Continuamente trato de cuestionar mi trabajo, intento hacerlos (sic) bajo una objetividad, que sé, no tendré, pero trato de cuestionarme anteponiendo a la soberbia, me pregunto qué hice, qué no hice, cómo fue mi funcionamiento y comparo con el ambiente del salón, para poder sacar una síntesis de lo hecho (Mendoza, 2012, p. 2).

Una condición, que provoca la reflexión natural en los docentes sometidos a un proceso sistematizado de formación continua, es el reconocimiento de la convicción como guía del trabajo de educar, ya sea que se convenció durante el proceso de reflexión o era ya un elemento permanente en la historia personal del docente. En el primer caso, se encuentran expresiones como "mejor trabajo no pude haber encontrado", debido a la satisfacción y crecimiento personal (Mendoza, 2012, p. 2); en el segundo caso, una docente asegura:

[...] estoy en la docencia por gusto, no porque el destino o las circunstancias me hayan traído aquí. Esta es una labor que disfruto hacer y que por consecuencia me compromete a hacer mi trabajo lo mejor que me sea posible, analizando mi práctica, innovando con y para mis estudiantes y compartiendo con mis compañeros docentes todo aquello que me funciona y que ellos estén abiertos a analizar. (Pliego; 2012, p. 5)

Con base en esa declaración y a partir de la guía de formación que imponen las actividades del programa de formación docente, la estudiante se torna capaz de reconocer la dimensión, características y consecuencias de la reflexión docente en los siguientes términos: 
La reflexión y la sistematización sobre la propia práctica docente es la herramienta que poseen los maestros para avanzar y superarse profesionalmente. No existe reflexión sin compromiso social, sin una reflexión crítica, sistemática y organizada. Sin reflexión y análisis permanentes, la práctica se mecaniza, se hace rutina y se empobrece. Librada a su propia inercia, la práctica puede enseñar poco, puede enseñar mal, puede enseñar a repetir los mismos errores, paralizarse en conocimientos desactualizados y en una vieja pedagogía impermeable a la experimentación y al cambio. (Pliego; 2012, p. 5)

Se puede observar que la docencia por vocación crea las condiciones para el pensamiento profundo que incluye no sólo la conceptualización de la reflexión como una actividad básica para la transformación cualitativa, sino el reconocimiento claro de las consecuencias negativas de permanecer ajeno y pasivo del contexto en el que se desarrolla la práctica educativa. Así pues, educar por convencimiento tiene sus resultados en la asunción de un compromiso claro con los estudiantes y hasta en la adopción de una postura crítica desde la cual es posible señalar las limitaciones, por ejemplo, de las políticas educativas y el alcance de su impacto. En la primera situación encontramos la reflexión de Romero (2012), quien señala lo siguiente:

Desde la mirada de ser docente por convicción resulta tan extraordinario reconocer que educar compromete, representa la posibilidad de transformar con base en elementos socioculturales vinculados con la práctica, implica responder al contexto, incorporar en el estudiante competencias para que logre desempeñarse eficaz y eficientemente en los medios de producción, ser emprendedor, creativo, crítico, analítico y sin duda que resuelva problemas de la vida diaria. (p. 1)

Si en el comentario anterior se reconoce el compromiso que nace en los docentes para transformarse de acuerdo al nuevo discurso educativo e inscribir sus esfuerzos hacia la formación de sus estudiantes, en otros, la preocupación se centra apenas en tratar de comprender los conceptos que componen la nueva filosofía de la educación por competencias como modelo educativo vigente; así llegan a declarar que "los docentes se encuentran más centrados en tratar de comprender el enfoque por competencias, que en el reconocimiento de las competencias que poseen y de las competencias que deben desarrollar para lograr darle sentido a la labor que desempeñan”(Juárez, 2012, p. 2).

Además del esfuerzo intelectual que implica comprender un modelo pedagógico antónimo a la formación y experiencia de los docentes de bachillerato, también se encuentra latente una postura crítica sobre el contexto que determina la práctica, en la cual se logra percibir la reflexión un tanto compleja. Por ejemplo, Juárez (2012) se plantea la siguiente ¿por qué hablar de competencias? y responderse a continuación que el cuestionamiento obliga a buscar conocimiento sobre las tendencias educativas mundiales que buscan "formar estudiantes capaces de enfrentar los cambios vertiginosos derivados de la sociedad de la información y el conocimiento y asídar respuesta a los retos que emanan de ésta (sic) sociedad"(p. 1); pero, también reconoce que los docentes no pueden estar exentos del desarrollo de sus propias competencias.

Destacar, en el origen de las reformas educativas locales, las tendencias mundiales no proyecta una visión simple o ingenua, pues el mismo estudiante argumenta que, por un lado, la 
negativa a reflexionar a la luz de un proyecto distinto mantiene la práctica educativa obsoleta y, por el otro, que la impronta de una reforma curricular y sus repercusiones en el trabajo docente tiene su origen en la globalización y las políticas neoliberales. Inclusive, entre el pensamiento generado, Díaz (2012) observa lo siguiente respecto de la enseñanza crítica-reflexiva:

Permite analizar, pensar, meditar, criticar y reorientar la práctica docente; además permite realizar una constante auto-evaluación que cuestione el desempeño, viendo actividades positivas y negativas, con el fin de facilitar el proceso enseñanza-aprendizaje. Reflexionar constantemente sobre nuestra práctica educativa, nos permite observar de qué manera estamos trabajando con los estudiantes, cómo es nuestra relación con los compañeros de trabajo y padres de familia. (Díaz, 2012, p. 2)

Sin embargo, otra postura expresada por un estudiante de la Maestría en Práctica Educativa indica que el modelo de profesor reflexivo puede ser un concepto más implícito en el enfoque educativo por competencias. Esto, toda vez que los docentes aceptan que los hechos de pensar con la finalidad de desarrollar el pensamiento crítico son positivos y que traerán en consecuencia, beneficios para las comunidades escolares; sin embargo, lo que no se deriva directamente del modelo educativo vigente son los procesos de cómo lograrlos. En este sentido, Díaz (2012) señala que:

Las supuestas ganancias pueden ser aparentes si sólo pueden generar procesos de discusión sobre el cómo de la práctica educativa, sin capacidad de decisión sobre los quéy los hacia dónde, que tienen que ser aceptados, o sin capacidad de intervención sobre las condiciones en las que transcurre la escolaridad. (Díaz, 2012, p. 2)

La reflexión anterior es profunda porque pone sobre la mesa la necesidad de que la reflexión docente también considere la función social de la práctica educativa y el interés político que se encuentra implícito en la reforma curricular de educación media superior, pues sólo bajo una mirada más amplia y profesionalizada del docente, el acto educativo se puede considerar más que un trabajo técnico. Y agrega:

Es necesario ubicarnos en el contexto global y complejo en el que se desenvuelve la educación basada en competencias. Así como también es importante observar cómo estas circunstancias exigen del profesional reflexivo la toma de consciencia para desarrollar competencias profesionales como vía exclusiva para alcanzar la eficiencia y la calidad educativa que exigen los nuevos tiempos. Dos aspectos contextuales, la posmodernidad como proyecto y el neoliberalismo como sistema económico, representan causalidades a considerar al tratar de comprender el surgimiento y desarrollo de la docencia reflexiva como un enfoque distinto al tradicional y al racional técnico. (Díaz, 2012, p. 1)

Es claro, entonces, que el perfeccionamiento de la práctica docente consiste en aprender metódicamente de la propia experiencia profesional mediante la reflexión sobre las prácticas, explicando principios y teorías que antes parecían invisibles (Luchetti, 2008, p. 15). Este ejercicio se puede traducir como el develamiento permanente de aquellos conocimientos que 
si bien no eran desconocidos por el profesor, sí carecían de una denominación o terminología específica dentro del campo de la pedagogía.

En una actividad de foro interactivo, una profesora estudiante del programa de Maestría en Práctica Educativa del Nivel Medio Superior comentó lo siguiente:

Después de leer y revisar literatura relacionada con la enseñanza reflexiva y la investigaciónacción, me di cuenta de que algunos de los conceptos desarrollados por los autores yo ya los llevaba a cabo en mis salones de clase. Un ejemplo de ello, es el manejo de los registros, diarios o bitácoras donde escribo después de cada clase todas aquellas situaciones que, a mi parecer, debía de mejorar en beneficio de mis estudiantes. A pesar de que yo tenía mis bitácoras de clase, por falta de sistematización no sabía cómo darle seguimiento.

-Estudiante-

Alatorre (203) propone la constante búsqueda de respuestas que nutran, mejoren y fortalezcan la práctica en la teoría. Así como de manera inversa, se recomienda retomar elementos de la práctica significada y, por medio de la investigación, someterlos al escrutinio de propuestas teóricas para elaborar las propias y así participar en un proceso constante de transformación de la práctica educativa.

Para los docentes, esta reflexión crítica conlleva la adopción y desarrollo de una práctica educativa progresista, la cual parte de una concientización sobre los límites y fortalezas de su propia práctica. Un educador progresista reconoce que para que se realicen transformaciones profundas y radicales se requiere de su participación activa y dinámica como agentes centrales del cambio, comenzando por la identificación del grado de participación que tienen en la práctica educativa. El grado de participación se traduce en la responsabilidad social que implica el quehacer de los docentes.

De acuerdo con Pedroza (2011) este replanteamiento exige, por una parte, la re-significación de la práctica docente y, por otra, la participación democrática de todos y cada uno de los miembros de la comunidad educativa en los distintos procesos políticos que se derivan al interior. En este mismo sentido, Paulo Freire $(1996,1997)$ sugiere hacer una "reflexión crítica sobre educación y calidad que sea conducente a la educación para la calidad, la calidad para la educación, la educación y la calidad de vida" (p. 41).

En consecuencia, se sugiere pensar la práctica educativa desde una óptica crítica; la formación docente se puede interpretar en un primer momento como el reconocimiento del papel que cada docente juega dentro su contexto educativo. No es posible pensar la práctica educativa sin la previa concientización de los docentes sobre el entorno que los rodea y su posición respecto de éste. En un segundo momento, una vez que la práctica es significada por el docente, ésta debe buscar sus opiniones fundamentadas o bien teoría que respalda el quehacer de los docentes.

Para comenzar a fomentar una cultura de responsabilidad social en el campo educativo, es necesario que los educadores desde una pedagogía crítica estén conscientes que, para un 
cambio radical en el orden educativo, primero se requiere de una transformación docente en el plano individual. Sin embargo, la identificación a partir de la reflexión sobre las debilidades y fortalezas de la práctica representa en sí el principal obstáculo para los docentes, quienes, desde una idea tradicional de la investigación enraizada profundamente, consideran que el problema de toda práctica educativa como posible objeto de estudio se encuentra fuera de ellos y, por tanto, su actividad docente no forma parte del problema. Lograr el auto-conocimiento a través de cuestionamientos, tales como qué es lo que hago, cómo lo hago, para qué lo hago y por qué lo hago, implican mucho más que una respuesta en el plano vertical de éstas, ya que conllevan el desarrollo de una nueva cultura entre los docentes: el desarrollo de una pedagogía crítica y auto-reflexiva.

El auto-conocimiento y la auto-exploración por parte de los docentes, sobre su práctica profesional, representan, de acuerdo con el diagnóstico que se presenta, el reto principal para los programas educativos de posgrado, cuyo objetivo es la profesionalización de la práctica docente. Probablemente, y como resultado de este acercamiento, una de las respuestas que permite explicar la razón de esta situación es que para el logro de una transformación educativa es necesario disminuir el distanciamiento que existe entre el discurso del docente y su práctica. Alatorre (2003) identifica que la práctica docente es un proceso complejo que se va construyendo al reflexionar sobre la acción o durante la acción. No se puede entender la generación de teoría educativa sin la participación activa de los educadores. La toma de decisiones, el diseño y planeación de políticas educativas requiere de la contribución de los docentes.

De acuerdo con las exigencias actuales, no se puede entender a la investigación educativa que se lleva a cabo desde fuera de las escuelas. Para ello, se necesita de una verdadera participación de los educadores en los distintos procesos de transformación. Lo anterior exige condiciones de reconocimiento del entorno donde se desarrolla la práctica educativa y de las distintas políticas educativas institucionales y nacionales; esto para lograr una comprensión y potencializar las oportunidades y fortalezas propias en el cumplimiento de las metas establecidas.

Un ejemplo de esos planteamientos lo constituyen los estudiantes de la Maestría en Práctica Educativa del Nivel Medio Superior de la UAEM, quienes en un esfuerzo por lograr la profesionalización de la práctica educativa tratan de romper los esquemas y estereotipos tradicionales del quehacer del profesor como investigador, en un primer momento. En un segundo momento, se observa que, al matricularse en un programa de estudios avanzados, cuyo objetivo es la profesionalización de la práctica educativa en el nivel medio superior, se responde al cambio en la cultura de quiénes enseñan, cómo enseñan y para qué enseñan; esto último se traduce en la reorientación de los fines de la educación en el nivel medio superior.

\section{Consideraciones finales}

Derivado de este acercamiento, un primer reto que enfrentan los programas, cuyo objetivo es la profesionalización de los docentes como el programa de Maestría en Práctica Educativa del Nivel Medio Superior, consiste en descartar toda posibilidad de preparar a los futuros o presentes docentes para un sistema educativo que ya no existe, porque el significado de su profesión habrá cambiado para cuando culminen con sus estudios. Por lo tanto, se deberá 
pensar en un replanteamiento en la naturaleza curricular de los estudios de posgrado que incluyan modelos flexibles que los docentes puedan traducir en formas adaptables de pensar sobre su práctica en momentos históricos diferentes para responder a las exigencias, reformas y transformaciones que les sean solicitados. En palabras de Luchetti (2008) la tarea de los docentes consiste en descubrir la nueva fisonomía de su papel, a lo que se le debe sumar las nuevas fisonomías cambiantes y demandantes del docente.

Un segundo reto consiste en reconocer que, para que la práctica educativa se perfeccione, es necesario que se someta al permanente análisis crítico del cual se desprenden la re-significación y la significación de la propia práctica docente. Tal y como se apunta en las primeras páginas de este documento, no es posible el cambio educativo sin la participación abierta y democrática de los profesores. Pensar su acción docente para practicar mejor representa uno de los retos más sobresalientes en este tipo de programas de estudio orientados hacia la profesionalización de la docencia.

Un tercer reto está representado por la imperiosa necesidad de posicionar a la práctica docente como pieza clave para la transformación social, si bien no constituye en sí misma el único componente para el cambio social, sí es una parte fundamental para la formación de las presentes generaciones. Sin embargo, se debe considerar que es necesario abandonar ideales impuestos por distintos medios de comunicación masivos en los que se posiciona a la educación como el único camino hacia la transformación social y el desarrollo de una nación, ya que, lejos de abonar a la evolución de una nueva cultura de formación de los sujetos, ésta se obstaculiza con imposición de responsabilidades sociales que no puede resolver.

Finalmente, con el afán de asumir la necesidad de reflexionar la práctica educativa como parte inicial del proceso para la formación de los nuevos cuadros de docentes que permita garantizar la calidad educativa, es necesaria la instrumentación de estrategias de formación continua y permanente que se sumen al proyecto educativo de los pueblos. La universidad e instituciones de educación superior formadoras de educadores juegan un papel fundamental en la reconstrucción y generación de políticas públicas, las cuales se traducen en políticas educativas. En este sentido, los programas educativos de calidad deben estar orientados hacia las necesidades y cambios que exige nuestra sociedad en materia educativa. Por esta razón, los programas de estudio con pertinencia social como el programa de Maestría en Práctica Educativa del Nivel Medio Superior representan una ventana de oportunidad para repensar la práctica educativa y la práctica docente como objetos de estudio, cuyos resultados conduzcan a la mejora de las condiciones actuales del proceso enseñanza-aprendizaje y, por tanto, reditúen en la calidad educativa.

\section{Referencias}

Alatorre, A. (2003). La investigación-acción: Conocer y cambiar la práctica educativa. Madrid: Graó.

Borg, S. (Primavera, 1998). Teachers' Pedagogical Systems and Grammar Teaching: A Qualitative Study [Los sistemas pedagógicos y enseñanza de la gramática de los maestros: Un Estudio Cualitativo]. TESOL Quartely, 32 (1), pp. 9-38. Recuperado de http://www.education.leeds.ac.uk/assets/files/staff/papers/Borg-TQ-32-1.pdf 
Cruz L. L. (2012). Conceptualización de las competencias docentes. En Comunidad: Forma ción basado en competencias, Módulo 4: La formación del profesor reflexivo, [Material Didáctico Publicado en la plataformaeducativa], Plataforma Seduca2/UAEMéx. Recuperado de http:// www.seduca2.uaemex.mx/distancia/Portafolio/portafolio.php?iCom=10388\&iInt=136314\&iCveEst=

Díaz, S. J. (2012), Conceptualización de las competencias docentes. En Comunidad: Formación basada en competencias, Módulo 4: La formación del profesor reflexivo, [Material Didáctico Publicado en la plataforma educativa], Plataforma Seduca2/UAEMéx. Recuperado de http:// www.seduca2.uaemex.mx/distancia/Portafolio/portafolio.php?iCom=10388\&iInt=136314\&iCveEst=

Erickson, F. (1997). Métodos cualitativos de investigación sobre la enseñanza. En M. Wittrock, La investigación de la enseñanza, II. Métodos cualitativos y de observación. Barcelona: Paidós.

Freire, P. (1996). Política y educación. México: Siglo XXI.

Freire, P. (1997). Pedagogía de la autonomía: Saberes necesarios para la práctica educativa. México, DF: Siglo XXI.

Freire, P. (2009). La educación como práctica de la libertad. México: Siglo XXI.

Gasca, E. (2012). Cuarto Informe de Actividades. Toluca, Universidad Autónoma del Estado de México. Recuperado de http://www.uaemex.mx/cuartoinforme/4toINFORME_WEB/ fscommand/Informe2012.pdf

García E.A.R.(2012). Ensayo. En Comunidad: Metodología de la intervención educativa,Módulo 1. ¿Quées la práctica educativa?, [Material Didáctico Publicado en la plataforma educativa], Plataforma Seduca2/UAEMéx. Recuperado de http://www.seduca2.uaemex. $\underline{\mathrm{mx} / \text { distancia/Portafolio/portafolio.php?iCom=10388\&iInt=136314\&iCveEst= }}$

Giroux, H. (1990). Los profesores como intelectuales. Hacia una pedagogía crítica del aprendizaje. Barcelona: Paidós. Recuperado de http://www.scribd.com/doc/104079547/Losprofesores-como-intelectuales-Hacia-una-pedagogia-critica-del-aprendizaje-Henry-Giroux

Juárez S. (2012). Reflexión sobre mi práctica educativa”. En Comunidad: Formación basada en competencias, Módulo 4: La formación del profesor reflexivo, [Material Didácti co Publicado en la plataforma educativa], Plataforma Seduca2, /UAEMéx. Recuperado de http://www.seduca2.uaemex.mx/distancia/Portafolio/portafolio.php?iCom $=10388 \&$ int $=136314 \& i$ CveEst=

Luchetti, E. (2008). Guía para la formación de nuevos docentes. Buenos Aires: Bonum. 
Macedo O. A. I. (2012). Importancia de la relación entre la teoría y la práctica educativa. En Comunidad: Metodología de la intervención educativa, Módulo 1. ¿Quées la práctica educativa?, [Material DidácticoPublicado en la plataformaeducativa], Plataforma Seduca2, /UAEMéx. Recuperado de http://www.seduca2.uaemex.mx/distancia/Portafolio/ portafolio.php?iCom=10388\&int=136314\&iCveEst=

Mendoza G. C. (2012). Reflexión sobre mi práctica educativa. En Comunidad: Formación basada en competencias, Módulo 4: La formación del profesor reflexivo, [Material Didáctico Publicado en la plataforma educativa], Plataforma Seduca2/UAEMéx. Recuperado de http://www.seduca2.uaemex.mx/distancia/Portafolio/portafolio.php?iCom $=10388 \& \mathrm{iInt}=136314 \& \mathrm{iCveEst}=$

McEwan, H; y K. Egan (Comps). (1998). La narrativa en la enseñanza, el aprendizaje y la investigación. Buenos Aires: Amorrortu. Recuperado de http://www.cep.edu.uy/ documentos/2014/aprender/NARRATIVA_MC_EWAN_EGAN.pdf

Meza, G. (2002). La teoría en la práctica educativa. Revista Comunicación. 12 (2), pp. 1-9. Recuperado de http://www.tec-digital.itcr.ac.cr/servicios/ojs/index.php/comunicacion/ article/view/1200/1107

Narváez C. E., Cadena, C. S., (2009). La enseñanza de la lectura académica: Un objeto de formación docente. Lectura y vida: Revista latinoamericana de lectura, 30 (1), pp. 56-67. Recuperado de http://www.lecturayvida.fahce.unlp.edu.ar/numeros/a30n1/30_01_Narvaez.pdf

Pedroza, F. R. (2011). La investigación-acción en la práctica educativa reflexiva. México: Colegio de Investigadores en Ciencias de la Educación.

Pliego F. G. G. (2012). Ensayo. En Comunidad: Formación basada en competencias, Módulo 4: La formación del profesor reflexivo, [Material Didáctico Publicado en la plataforma educativa], Plataforma Seduca2/UAEMéx. Recuperado de http://www.seduca2.uaemex.

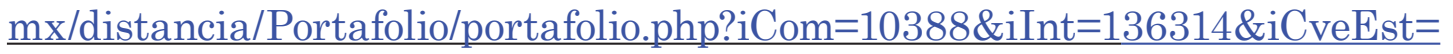

Pruzzo, V. (2002a). Transformación de la formación docente. Buenos. Aires: Espacio Editorial.

Pruzzo, V. (2002b). La formación del profesorado. Aportes críticos al dispositivo de análisis de clases (D.A.C.), Revista Horizontes Educacionales. 7(1), pp. 7-22. Universidad del BíoBío, Chillán, Chile. Recuperado de http://dialnet.unirioja.es/servlet/articulo?codigo=3994339

Romero, A. (2012). Ser docente competente: paradoja de este siglo. En Comunidad: Formación basada en competencias, Módulo 4: La formación del profesor reflexivo, [Material Didáctico Publicado en la plataforma educativa], Plataforma Seduca2, /UAEMéx. 
Recuperado de http://www.seduca2.uaemex.mx/distancia/Portafolio/portafolio.php?iCom $=10388 \&$ int $=136314 \&$ iCveEst $=$

Secretaría de Educación Pública (SEP). (2008), Acuerdo número 444 por el que se establecen las competencias que constituyen el marco curricular común del Sistema Nacional de Bachillerato, México. Recuperado de http://www.stunam.org.mx/sa/seccionacad/prepas/ acuerdo444.pdf

Universidad Autónoma del Estado de México (UAEM). (2010). Programa de Estudios de la Maestría en Práctica Educativa del Nivel Medio Superior. [Manuscrito sin publicar], Secretaría de Investigación y Estudios Avanzados, Universidad Autónoma del Estado de México, Toluca, México. 